\title{
Distance determination to NGC 55 from the planetary nebula luminosity function ${ }^{\star}$
}

\author{
G. C. Van de Steene ${ }^{1}$, G. H. Jacoby ${ }^{2}$, C. Praet $^{3}$, R. Ciardullo ${ }^{4}$, and H. Dejonghe ${ }^{3}$ \\ 1 Royal Observatory of Belgium, Ringlaan 3, 1180 Brussels, Belgium \\ e-mail: gsteene@oma . be \\ 2 WIYN Observatory, PO Box 26732, Tucson, AZ, 85726, USA \\ e-mail: gjacoby@noao.edu \\ 3 Sterrenkundig Observatorium, Krijgslaan 281, 9000 Gent, Belgium \\ e-mail: H.Dejonghe@rug.ac.be \\ 4 Department of Astronomy and Astrophysics, Penn State University, 525 Davey Lab University Park, PA 16802, USA \\ e-mail: rbc@astro.psu.edu
}

Received 19 May 2005 / Accepted 17 May 2006

\section{ABSTRACT}

We analysed [O III], $\mathrm{H} \alpha$, and continuum images of the Sculptor Group spiral galaxy NGC 55 obtained with the WFI instrument at the 2.2-m telescope of ESO. We identified 21 new planetary nebula candidates. We constructed the [O III] $\lambda 5007$ Planetary Nebula Luminosity Function (PNLF) and determined a most likely distance of $2.30 \pm 0.35 \mathrm{Mpc}$. The distance to NGC 55 is a bit larger than previously determined distances, which means that the Sculptor Group is further away from the Local Group than previously thought. The PNLF distance to NGC 55 is comparable to the PNLF distance of NGC 300 (Soffner et al. 1996, A\&A, 306, 9), adding support to the suggestion that these galaxies form a bound pair. There doesn't seem to be a shortage of planetary nebula candidates in this metal poor galaxy.

Key words. planetary nebulae: general - galaxies: individual: NGC 55 - galaxies: distances and redshifts galaxies: clusters: individual: Sculptor Group

\section{Introduction}

Planetary Nebulae (PNe) are bright emission line objects, observable out to very large distances. Numerous PNe in many other galaxies have been detected. They are excellent probes of the chemical enrichment history of a galaxy (Dopita et al. 1997; Walsh et al. 2000; Magrini et al. 2004) and of galactic dynamics (Mathieu et al. 1996; Durand et al. 1996; Ciardullo et al. 2004; Napolitano et al. 2001).

The bright end of the PN Luminosity Function (PNLF) has been empirically shown to be invariant enough to serve as a secondary standard candle for extra-galactic distance determinations (Jacoby et al. 1992; Jacoby \& Ciardullo 1993). It has been carefully calibrated (Ciardullo 2003). It shows good internal consistency, and good external agreement.

NGC 55 is a highly inclined late type galaxy in the Sculptor Group. Its properties are listed in Table 1. Sculptor is a very loosely concentrated and almost freely expanding aggregate of galaxies of prolate shape which we view pole-on. It stretches from the outskirts of the Local Group out at about $1.5 \mathrm{Mpc}$ to $6 \mathrm{Mpc}$. Apparently, the near and the far parts are not gravitationally bound to each other, but take part in the general Hubble flow. The galaxy complexes in Sculptor and Canes Venatici together with the Local Group form a filament extending over $\pm 10 \mathrm{Mpc}$. NGC 55 is one of the 5 bright spiral galaxies of the Sculptor Group. Besides the 5 main galaxies, the Sculptor Group contains several dwarf galaxies (Jerjen et al. 1998, 2000; Karachentsev et al. 2003). Distance estimates for

${ }^{\star}$ Based on observations collected at the European Southern Observatory, Chile.
Table 1. Basic properties of NGC 55.

\begin{tabular}{rll}
\hline \hline RA (J2000) & $00^{\mathrm{h}} 15^{\mathrm{m}} 8^{\mathrm{s} .5}$ & 1 \\
Dec (J2000) & $-39^{\circ} 13^{\prime} 13^{\prime \prime}$ & 1 \\
Type & SB $(\mathrm{s}) \mathrm{m}$ & 2 \\
$E(B-V)$ & $0.013 \mathrm{mag}$ & 3 \\
$V_{\text {helio }}$ & $116 \pm 2 \mathrm{~km} \mathrm{~s}^{-1}$ & 3 \\
$\mathrm{i}$ & $85 \pm 5^{\circ}$ & 3 \\
$R_{25}$ & $18^{\prime} .9=8.8 \mathrm{kpc}$ & 2 \\
$R_{\mathrm{Holm}}$ & $20.2=9.5 \mathrm{kpc}$ & 2 \\
$m_{B}$ & 8.10 & 4 \\
\hline
\end{tabular}

(1) Third Reference Catalogue of bright galaxies (RC3).

(2) NASA Extragalactic Database.

(3) Schlegel et al. (1998).

(4) Giuricin et al. (2000).

NGC 55 range from 1.34 Mpc based on carbon stars (Pritchet et al. 1987) to $1.8 \mathrm{Mpc}$ based on the revised Tully-Fisher relation (Karachentsev et al. 2003), The two bright spiral galaxies NGC 55 and NGC 300 are both at the near side of the Sculptor Group and it has been suggested that NGC 55 and NGC 300 form a bound pair (Graham 1982; Pritchet et al. 1987; Whiting 1999). The distance to NGC 300 is well determined via Cepheids (Freedman et al. 1992: 2.1 $\pm 0.1 \mathrm{Mpc}$ ) and via the PNLF (Soffner et al. 1996: $2.4 \pm 0.4 \mathrm{Mpc}$ ). NGC 55 has remained without a reliable distance estimate.

In Sect. 2 we discuss the observations, in Sect. 3 the data reduction, and in Sect. 4 the data analysis and results. The PNLF distance to NGC 55 is derived in Sect. 5, and some related issues are discussed in Sect. 6. 
Table 2. Observing log of the WFI observations of NGC 55. The first column gives the ESO filter-name, the second the central wavelength, the third the filter-width, the fourth the date of the observations, the fifth column the total integration time in seconds, and the last column the number of exposures.

\begin{tabular}{lrrrrr}
\hline \hline Filter & $\lambda$ & $\Delta \lambda$ & Date & I.T. & $N$ \\
name & $\AA$ & $\AA$ & $\mathrm{dd} / \mathrm{mm} / \mathrm{yy}$ & $\mathrm{s}$ & \\
\hline OIII/8 & 5023.93 & 80.34 & $04 / 07 / 99$ & 1800 & 3 \\
& & & $05 / 07 / 99$ & 1320 & 3 \\
$518 / 16$ & 5188.29 & 163.45 & $04 / 07 / 99$ & 900 & 3 \\
& & & $05 / 07 / 99$ & 720 & 4 \\
$\mathrm{H} \alpha / 7$ & \multirow{2}{*}{5888.27} & 74.31 & $04 / 07 / 99$ & 1800 & 3 \\
& & & $05 / 07 / 99$ & 1800 & 3 \\
\hline
\end{tabular}

\section{Observations}

The observations were done with the Wide Field Imager (WFI) on the 2.2-m telescope at the La Silla Observatory of the European Southern Observatory on the 4 and 5 July 1999. The weather was photometric. WFI consists of a 4 times 2 mosaic of $2 \mathrm{k} \times 4 \mathrm{k}$ CCDs. The pixel scale is 0.238 and the total field of view is $34^{\prime} \times 33^{\prime}$, which covers the entire galaxy. NGC 55 was observed through the [O III] and the off-band filter 518/16, and the $\mathrm{H} \alpha$ filter for a total of $3120 \mathrm{~s}, 1620 \mathrm{~s}$, and $3600 \mathrm{~s}$ respectively. On the first day the galaxy was offsetted to the east and south by $3^{\prime}$ to fill in the CCD gaps. The observing log is presented in Table 2 . The seeing was $\sim 11^{\prime \prime} 4$.

\section{Data reduction}

The ESOWFI external package in IRAF was used for converting the ESO headers to work with the MSCRED package. It also sets the default instrument files and an astrometry solution. Next the reductions were done using the MSCRED mosaic reduction package in IRAF according to the guide by Valdes (1998).

The data were bias substracted and flatfielded using twilight skyflats. These multiple CCD images and multiple, dithered, exposures were combined into a single image. To this end we accurately registered the images, made a mosaic of the exposures into single images with the same spatial sampling, measured changes in the intensity scale due to variations in transparency and sky brightness, and combined the individual images into a single deep image. These steps were applied to the [O III] on-band and off-band images, and the $\mathrm{H} \alpha$ images.

\section{Data analysis and results}

We followed the survey technique as described in e.g. Jacoby et al. (1989b) and Ciardullo et al. (1989a). The on- and off-band images were aligned. The off-band images were then scaled to the level of the on-band images and subtracted from them to produce the difference image. We identified the PNe by blinking this difference image with the off-band image, and the $\mathrm{H} \alpha+[\mathrm{N} \mathrm{II}]$ images. In order to discriminate PNe from other emission-line sources, we used the following criteria (Feldmeier et al. 1997): (1) PN candidates had to have a point-spread function (PSF) consistent with that of a point source as all PNe are expected to be unresolved at the distance of NGC 55; (2) PN candidates had to be invisible on the off-band image to exclude bright OB stars exciting H II regions; and (3) PN candidates had to be significantly fainter in $\mathrm{H} \alpha+[\mathrm{N} \mathrm{II}]$ image than [O III], in order to reduce further the possibility of contamination from
$\mathrm{H}$ II regions. With all these constraints, only compact, H II regions that have high nebula excitation and faint central OB associations will have been mistaken for PNe. However, even these will be faint and hence not significantly affect the PNLF.

In Table 3 we list our 21 newly identified PN candidates.

The plate solutions were created using reference stars from the FK5 (30 stars) and USNO-A.2.0 Astrometric Catalog (1370 stars) (Monet et al. 1998). The error in the equatorial coordinates are subarcsecond. No PNe are found very close to the centre and in the disk, because of numerous extended H II regions that cover the plane and bulge. PN candidates are found all along the galaxy. They are all within the gaseous H I envelope (Puche et al. 1991), just outside the brightest H I disk $\left(45^{\prime} \times 12^{\prime}\right)$. Only $4 \mathrm{PN}$ candidates are located south of the H I disk, the rest is located towards the north.

The PN candidates were measured photometrically in the off-band image using PHOT in IRAF and flux calibrated using standard stars and the procedures outlined in Jacoby et al. (1987). Because of the heavy crowding in the fields, aperture photometry was performed on the difference image. This image is more immune to confusion due to stars than the on-band image and the flux values are automatically corrected for the presence of continuum emission. In order to determine the filter transmission for the PNe we need to take the redshift into account and the bandpass shift of the interference filter to the blue. The systemic velocity of NGC 55 was taken to be $116 \mathrm{~km} \mathrm{~s}^{-1}$ (Puche \& Carignan 1991). Hence, the redshift of the [O III] and $\mathrm{H} \alpha$ emission lines of the PNe in NGC 55 are respectively $1.936 \AA$ and $2.538 \AA$.

From the ESO web pages we obtained the filter transmission curves and the standard star spectra. The incident angle of the beam with the filter causes a change in transmission across the filter in the sense that the peak central wavelength of the filter shifts bluewards by about $0.1 \%$ at the centre of the filter to $0.2 \%$ at the edge of the filter. All these effects cause the [O III] line to be closer towards the peak transmission of the filter. The percentage of transmission is about $88 \%$ across the filter for the [O III] line and about $87 \%$ for the $\mathrm{H} \alpha$ filter at the location of our objects. The difference in transmission across the field has negligible effect on the derived flux values and magnitudes. The resulting filter transmission curve of each filter was convolved with the spectral-energy distribution of each standard star in IDL to obtain their flux values within each filter passband. The total number of counts obtained from the photometry of the WFI image of the standard star was related to these absolute flux values to derive the total system sensitivity for each standard star (Table 4). The average of the obtained values was adopted to derive the absolute flux values for the PNe and given in Table 5 with other parameters used in the flux calibration.

The resulting monochromatic [O III] flux values $F_{5007}$ were converted to [O III] $\lambda 5007$ magnitudes using (Jacoby 1989):

$m_{5007}=-2.5 \log \left(F_{5007}\right)-13.74$.

The errors listed in 5 are the measurement error as given by the PHOT program in IRAF. To this we need to add the systematic errors such as the uncertainty in the zero point, the foreground extinction, the system sensitivity, the aperture correction, the filter response curve etc. These errors are the same for all PNe. To take them into account we added $0.1 \mathrm{mag}$ to the photometric errors listed in Table 5.

If the ratio of $F_{5007}$ to $F_{\mathrm{H}_{\alpha}}$ is at least 3 to 1 , then it is very likely to be a PN rather than an HII region, because of the excitation levels. To produce an HII region with strong 
Table 3. The first column gives the number of the PN, the second and third the $(x, y)$ pixel coordinates, the fourth and fifth the RA and Dec J2000.0 coordinates, the sixth column the number of counts in adu. The 7th column gives the [O III] flux values after correction for the filter transmission, the 8 th column the $[\mathrm{O} \mathrm{III}]$ magnitudes, and the last column the error as given by the PHOT program.

\begin{tabular}{|c|c|c|c|c|c|c|c|c|}
\hline & $\begin{array}{l}X \\
\text { pixels }\end{array}$ & $\begin{array}{l}Y \\
\text { pixels }\end{array}$ & $\begin{array}{l}\text { RA (J2000) } \\
\text { h m s }\end{array}$ & $\begin{array}{l}\overline{D e c}(\mathrm{~J} 2000) \\
{ }^{\prime}, \prime \prime\end{array}$ & $\begin{array}{l}\text { Counts } \\
\text { adu }\end{array}$ & $\begin{array}{l}F_{5007} \\
10^{-15} \mathrm{erg} \mathrm{s}^{-1} \mathrm{~cm}^{-2}\end{array}$ & $\begin{array}{l}m_{5007} \\
\mathrm{mag}\end{array}$ & $\begin{array}{l}\text { err } \\
\text { mag }\end{array}$ \\
\hline \multicolumn{9}{|c|}{ [O III] (I.T. $600 \mathrm{~s}$ ) (Airmass 1.073) } \\
\hline 1 & 7269.9 & 4756.7 & 001424.37 & -390734.3 & 1387.872 & 1.79853 & 23.123 & 0.055 \\
\hline 2 & 6095.9 & 4671.5 & 001448.36 & -390755.7 & 962.394 & 1.24716 & 23.520 & 0.060 \\
\hline 3 & 5182.4 & 4104.6 & 001507.02 & -301011.2 & 916.6378 & 1.18786 & 23.573 & 0.058 \\
\hline 4 & 5112.9 & 3095.1 & 001508.39 & -391411.4 & 830.136 & 1.07576 & 23.681 & 0.063 \\
\hline 5 & 5126.0 & 4726.6 & 001508.20 & -390743.2 & 1364.671 & 1.76846 & 23.141 & 0.041 \\
\hline 6 & 5051.6 & 3957.7 & 001509.68 & -391046.2 & 1031.522 & 1.33674 & 23.445 & 0.069 \\
\hline 7 & 4755.9 & 3889.7 & 001515.73 & -391102.6 & 1240.388 & 1.60741 & 23.245 & 0.054 \\
\hline 8 & 4707.4 & 3154.1 & 001516.70 & -391357.6 & 890.097 & 1.15347 & 23.605 & 0.070 \\
\hline 9 & 4492.4 & 3911.3 & 001521.12 & -391057.5 & 981.333 & 1.27170 & 23.499 & 0.051 \\
\hline 10 & 4087.1 & 3773.6 & 001529.41 & -391130.4 & 1705.006 & 2.20950 & 22.899 & 0.033 \\
\hline 11 & 3971.1 & 3541.2 & 001531.78 & $\begin{array}{lll}-39 & 125.8\end{array}$ & 1474.990 & 1.91142 & 23.057 & 0.042 \\
\hline 12 & 3819.2 & 3566.5 & 001534.89 & -39 1219.8 & 1880.293 & 2.43665 & 22.793 & 0.035 \\
\hline 13 & 3741.2 & 3340.2 & 001536.48 & $\begin{array}{lll}-39 & 13 & 13.7\end{array}$ & 1316.036 & 1.70544 & 23.180 & 0.047 \\
\hline 14 & 3534.8 & 2288.6 & 001540.69 & -391723.9 & 2253.842 & 2.92073 & 22.596 & 0.027 \\
\hline 15 & 2974.1 & 2408.9 & 001552.18 & -391655.4 & 1198.998 & 1.55377 & 23.282 & 0.053 \\
\hline 16 & 2935.6 & 3228.8 & 001552.97 & -391340.3 & 1019.351 & 1.32097 & 23.458 & 0.063 \\
\hline 17 & 2832.6 & 3397.6 & 001555.08 & -391300.2 & 1616.177 & 2.09439 & 22.958 & 0.042 \\
\hline 18 & 2598.4 & 3493.3 & 001559.88 & -391237.4 & 1333.024 & 1.72745 & 23.167 & 0.047 \\
\hline 19 & 2009.2 & 3474.2 & 001611.93 & -391241.9 & 1004.969 & 1.30233 & 23.473 & 0.061 \\
\hline 20 & 1517.4 & 2625.8 & 001622.02 & -391603.7 & 1169.726 & 1.51584 & 23.308 & 0.045 \\
\hline 21 & 1909.1 & 2794.1 & 001614.00 & -39 1523.7 & 1714.469 & 2.22137 & 23.893 & 0.030 \\
\hline \multicolumn{9}{|c|}{$\mathrm{H} \alpha+[\mathrm{N} \mathrm{II}]$ (I.T. $600 \mathrm{~s})$ (Airmass 1.023$)$} \\
\hline 14 & 3521.6 & 2295.2 & 001540.69 & -39 1723.9 & 1095.789 & 1.0798 & & 0.066 \\
\hline 17 & 2819.2 & 3404.9 & 001555.08 & -391300.2 & 1028.824 & 1.0138 & & 0.072 \\
\hline 21 & 1895.1 & 2800.7 & 001614.00 & -391523.7 & 839.917 & 0.8277 & & 0.073 \\
\hline
\end{tabular}

Table 4. System sensitivity. The first column gives the name of the standard star, the second column its flux value, the third the number of counts per second, the fourth the airmass, and the last column the derived scaling factor.

\begin{tabular}{lllll}
\hline \hline Star & $\begin{array}{l}\text { Flux } \\
\mathrm{erg} \mathrm{s}^{-1} \mathrm{~cm}^{-2}\end{array}$ & $\begin{array}{l}\text { Cs } \\
\mathrm{adu} \mathrm{s}^{-1}\end{array}$ & Airmass & $\begin{array}{l}S \\
\mathrm{erg} \mathrm{cm} \mathrm{adu}^{-1}\end{array}$ \\
\hline [O III] & & & & \\
$\mathrm{ltt} 377$ & $9.42258 \times 10^{-12}$ & 14041.2867 & 1.005 & $5.63359 \times 10^{-16}$ \\
$\mathrm{ltt} 6248$ & $5.56021 \times 10^{-12}$ & 8017.7072 & 1.131 & $5.69557 \times 10^{-16}$ \\
$\mathrm{~cd}-32$ & $2.09332 \times 10^{-11}$ & 29915.4059 & 1.023 & $5.85601 \times 10^{-16}$ \\
eg274 & $1.38942 \times 10^{-11}$ & 20372.6927 & 1.214 & $5.52086 \times 10^{-16}$ \\
\hline $\mathrm{H} \alpha$ & & & & \\
$\mathrm{ltt} 377$ & $6.6890 \times 10^{-12}$ & 12625.6761 & 1.007 & $4.61467 \times 10^{-16}$ \\
$\mathrm{~cd}-32$ & $1.08421 \times 10^{-11}$ & 20843.8669 & 1.025 & $4.75044 \times 10^{-16}$ \\
eg274 & $4.41063 \times 10^{-12}$ & 8366.3110 & 1.222 & $4.73197 \times 10^{-16}$ \\
\hline
\end{tabular}

[O III] $] 5007$ flux, the ionizing star must be very hot and therefore very luminous, while on the main sequence. Hence these must be brighter than about $M_{V} \sim-6$, or apparent magnitude $m_{V}<21 \mathrm{mag}$ at a distance of $\sim 2.3 \mathrm{Mpc}$. We would have seen these in the off-band image, since the completeness limit is $m_{V} \sim 21.5 \mathrm{mag}$. None of the PN candidates were detected in the off-band image. Most show hints of $\mathrm{H} \alpha$ emission at the right position, but are too faint for flux determination. From Table 3 we see that 3 of our PN candidates, were detected in the continuum subtracted $\mathrm{H} \alpha+[\mathrm{NII}]$ image. Only number 17 has an [O III] to $\mathrm{H} \alpha+[\mathrm{NII}]$ flux ratio, which is about 2. As it doesn't show up in the off-band we leave it in the sample. (Note that if we had
Table 5. The average scaling factor, the atmospheric extinction, and the airmass used in the flux calculation.

\begin{tabular}{llll}
\hline \hline Filter & $\langle S\rangle$ & $k_{\lambda}$ & Airmass \\
\hline$[\mathrm{OIII}]$ & $5.67651 \times 10^{-16}$ & 0.189 & 1.073 \\
$\mathrm{H} \alpha$ & $4.6990 \times 10^{-16}$ & 0.096 & 1.023 \\
\hline
\end{tabular}

removed it from the sample the PNLF fit would get a little better and the distance a little further by only a few hundredths of magnitude, not significantly.) 


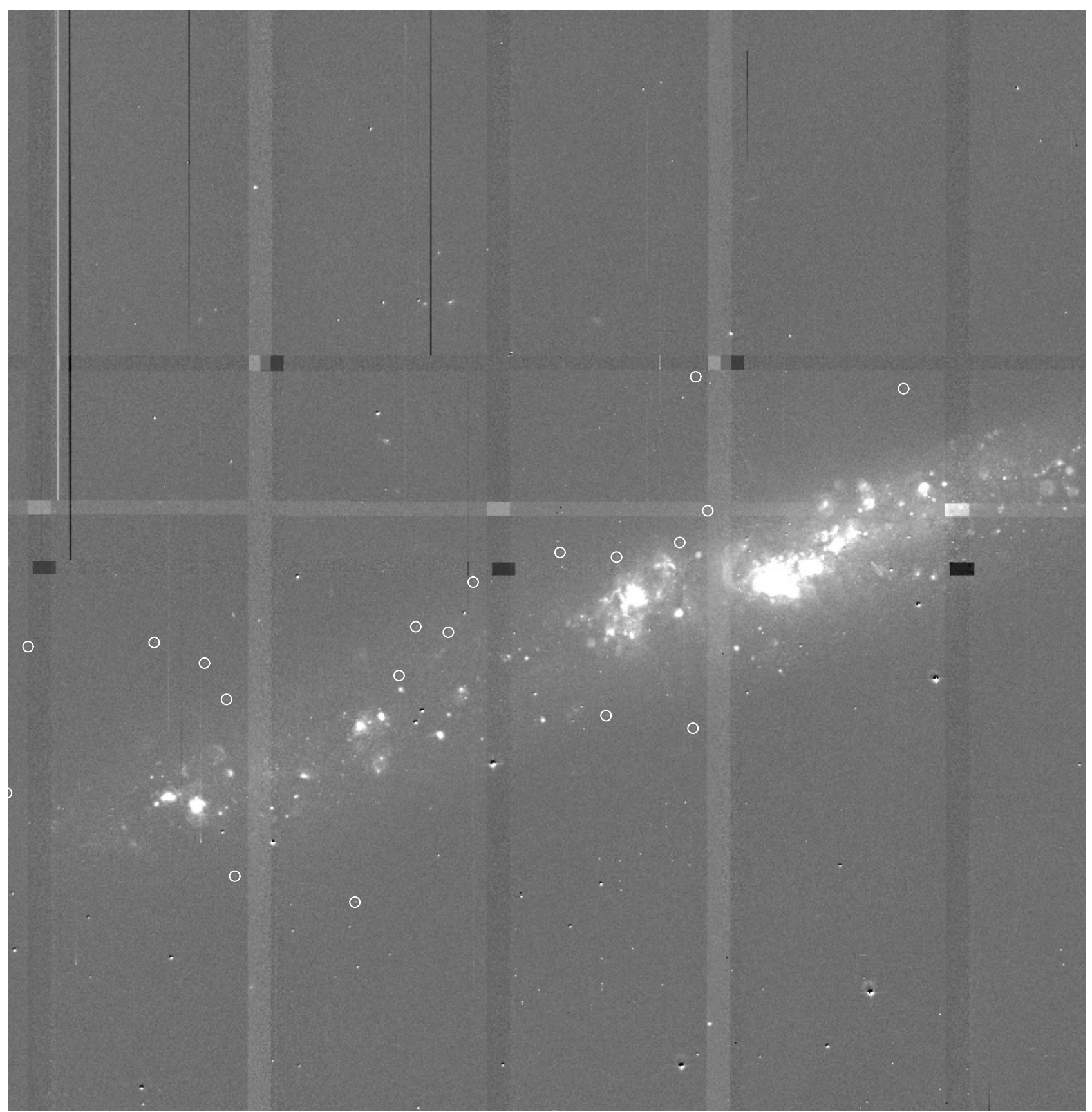

Fig. 1. PN candidates in the [O III $]$ continuum subtracted image of NGC 55. North is at the top and east to the left.

\section{The PNLF distance}

[O III] magnitudes were corrected for the interstellar extinction. We consider only the foreground Galactic extinction towards NGC 55 and adopt $E(B-V)=0.013$ mag (Schlegel et al. 1998). Identifications become incomplete beyond $\mathrm{m}_{5007}=23.5 \mathrm{mag}$. Besides missing faint $\mathrm{PNe}$, the probability of overlap with a star or with an $\mathrm{H}$ II region increases towards the centre, particularly in the $\mathrm{H} \alpha+[\mathrm{N} \mathrm{II}]$ image. Hence brighter PNe than the ones recovered in the outskirts may have been missed towards the center.

From these data the PNLF distance to the galaxy can normally be derived by convolving the empirical model for the PNLF given by Ciardullo et al. (1989b):

$N(m) \propto \mathrm{e}^{0.307 M}\left[1-\mathrm{e}^{3\left(M^{*}-M\right)}\right]$ with the photometric error function and fitting the data to the resultant curve via the method of maximum likelihood. This takes into account that the probability of observing PNe near the cutoff magnitude $M^{*}$ decreases for small sample sizes. Figure 2 plots the PNLF for the sample. Assuming $M^{*}=-4.47$, based on PNLF and Cepheid distance measurements of 13 galaxies (Ciardullo 2003), we obtain a most likely distance modulus $\left(m-M^{*}\right)=26.95 \pm 0.2 \mathrm{mag}$, which corresponds to a distance of $2.45 \pm 0.2 \mathrm{Mpc}$.

\section{Discussion}

\subsection{Metallicity}

The PNLF cut-off is fainter in small, low metallicity galaxies, but well modeled by the theoretical relation of Dopita et al. (1992). The metallicity correction as determined from the 


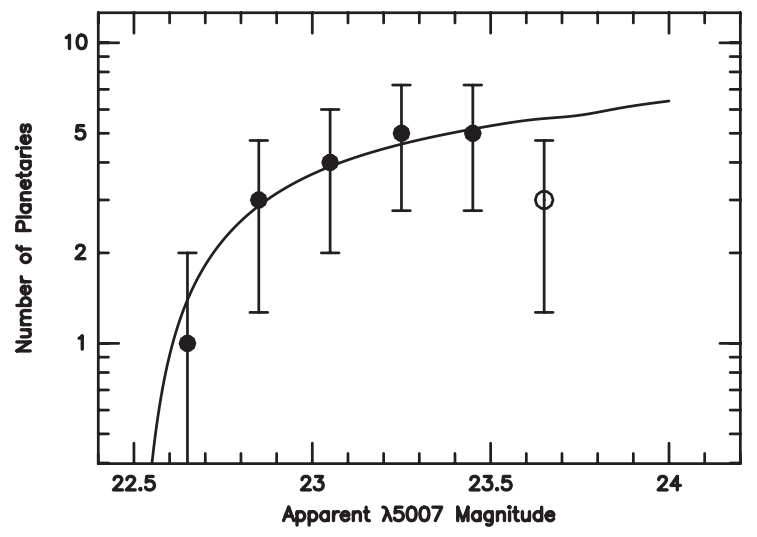

Fig. 2. Observed [O III] $] 5007$ PNLF of NGC 55. The curve represents the best-fitting empirical PNLF convolved with the photometric error function and shifted to the most likely distance. The open circle represents a point past the completeness limit.

oxygen abundance, is needed only for galaxies with metallicities smaller than the LMC $(12+\log (\mathrm{O} / \mathrm{H})<8.5)$ (Ciardullo et al. 2002). The oxygen abundance of NGC 55 as determined based on an $\mathrm{H}$ II region in the disk is $12+\log (\mathrm{O} / \mathrm{H})=8.05 \pm 0.1$ (Tüllmann et al. 2003). This value is similar to the SMC metallicity and hence the distance modulus needs to be corrected by $-0.48 \pm 0.15 \mathrm{mag}$. The metallicity corrected value of the distance modulus is $\left(m-M^{*}\right)=26.50 \pm 0.35$, which corresponds to a distance of $2.00 \pm 0.35 \mathrm{Mpc}$. The oxygen abundance determined by Tüllmann et al. (2003) with the VLT is the lowest ever. They average previously determined literature values to obtain a reference abundance in the disk of $12+\log (\mathrm{O} / \mathrm{H})=8.36$. This value is equal to the oxygen abundance of NGC 300 and would involve a correction to the distance modulus of only -0.15 mag (Ciardullo et al. 2002). In this case the metallicity corrected value of the distance modulus is $\left(m-M^{*}\right)=26.80 \pm 0.35$, which corresponds to a distance of $2.29 \pm 0.35 \mathrm{Mpc}$. The low metallicity of NGC 55 makes its PNLF distance somewhat dependent upon metallicity and consequently less certain. We adopt $2.30 \pm 0.35 \mathrm{Mpc}$ as the most likely distance to NGC 55.

NGC 300 also has a low metallicity, though not as low as NGC 55 , but still needs a metallicity correction by -0.15 mag (Ciardullo et al. 2002). The distance modulus to NGC 300 determined via the PNLF maximum likelihood method is 26.9 mag (Soffner et al. 1996) and after correction for metallicity $26.75 \mathrm{mag}$ or $2.24 \pm 0.4 \mathrm{Mpc}$, which is in good agreement with the Cepheid distance determination of $2.02 \pm 0.07 \mathrm{Mpc}$ by Freedman et al. (1992). The PNLF distances to NGC 55 and NGC 300 are again similar.

\subsection{Previous distance estimates}

Previous distance estimates to NGC 55 include $1.45 \mathrm{Mpc}$ based on the tip of the red giant branch method (Graham 1982), $1.34 \mathrm{Mpc}$ based on photometry of Carbon stars (Pritchet et al. 1987), 1.8 Mpc based on the revised Tuller-Fisher relationship (Karachentsev et al. 2003), and 2.12 $\pm 0.10 \mathrm{Mpc}$ based on the RGB population at large galactocentric radii (Tikhonov et al. 2004). Our distance determination agrees with the one from Tikhonov et al., but is a bit larger than previously determined distances. This means that the near end of the Sculptor Group is a bit further away than previously thought.

\subsection{Number of $P N e$}

It has been suggested that there is a slight tendency for the number of detected PNe to decrease with metallicity. There are some hints in the shortfall in the observed number of PNe $[\mathrm{Fe} / \mathrm{H}] \ll-1$, which corresponds to the point where the AGB wind is expected to be driven no longer by dust, but only by pulsations (Zijlstra 1999). Tikhonov et al. (2004) determined a mean metallicity of $[\mathrm{Fe} / \mathrm{H}]=-1.25$ for NGC 55 . Hence, the question is whether the number of $\mathrm{PN}$ candidates agree with the number of PNe one would expect to detect in NGC 55?

For a coeval and chemically homogeneous stellar population, the number of stars $n$ in any post-main sequence phase $j$ is given by the formula (Renzini \& Buzzoni 1986):

$n_{j}=\dot{\xi} L_{\mathrm{T}} t_{j}$

where $\dot{\xi}$ is the specific evolutionary flux (number of stars per unit luminosity leaving the main sequence each year), $L_{\mathrm{T}}$ the total luminosity of the sampled population, and $t_{j}$ the duration of the evolutionary phase.

The lifetime of a PN is typically $20000 \mathrm{yr}$.

The total number of PNe depends upon the bolometric luminosity of the galaxy. We adopted the UBV total corrected magnitudes $(7.74,7.63,7.09$ mag resp.) from the NED database, as well as the total 2MASS JHK flux values (6.976, 6.546, 6.249 mag resp.), with their corresponding flux values in Janskys. We adopted a total $R$ and $I$ magnitude of resp. 7.49 mag and $7.30 \mathrm{mag}$ (Fitzgibbons 1990). The SED obtained in this way was integrated to derive a bolometric luminosity of $3.9 \times 10^{9} L_{\odot}$.

The typical value of $\dot{\xi}$ is typically $1-2 \times 10^{-11} \mathrm{PNe}$ per year per solar luminosity (Renzini \& Buzzoni 1986).

Hence, the total number of PNe in NGC 55 would be between 750 and 1600. Because of the huge H II regions, we could not detect any PNe in the center of NGC 55. Based on the countour plots by Puche et al. (1991), we estimate that we surveyed only $20 \%$ of the luminosity. Hence the number of PNe we would expect to detect is between 100 and 350 . Based on the luminosity function we see that we only detected the brightest $\mathrm{PNe}$ in NGC 55. The [O III] luminosity of a PN declines rapidly once the nebula becomes optically thin, and the phase during which a PN is bright enough to be discovered is much shorter than the typical PN lifetime of $20000 \mathrm{yr}$. PNe are bright until they become optically thin at a radius of approximately $0.05 \mathrm{pc}$, which occurs after $2500 \mathrm{yr}$ for a nebular expansion velocity of $20 \mathrm{~km} \mathrm{~s}^{-1}$ (Magrini et al. 2003). Thus we would expect to find 18 to 40 bright PNe in NGC 55. Soffner et al. (1996) report a similar sampled luminosity and 10 PNe brighter than $m(5007)=24$ mag. From the 21 PNe found in NGC 55 we can expect its PN formation rate to be at least comparable to and possibly a bith highter than that of NGC 300. Although the uncertainty of these numbers is very large and all PNe candidates may not turn out to be true $\mathrm{PNe}$, we find no indication yet that there may be a lack of PNe in NGC 55.

\section{Conclusions}

We identified 21 new PNe candidates in the Sculptor Group galaxy NGC 55. The PNLF method gives us a most likely distance of $2.30 \pm 0.35 \mathrm{Mpc}$. It would mean that the Sculptor Group is a bit further away from the Local Group than previously thought. The distance to NGC 55 turns out to be comparable to the distance of NGC 300, adding support to the suggestion that these galaxies form a bound pair. We find no indication yet that there may be a lack of PNe in this low metallicity galaxy. 
Acknowledgements. G. C. Van de Steene acknowledges IUAP P5/36, financed by the OSTC of Belgium. This research has made use of the SIMBAD database, operated at CDS, Strasbourg, France and the NASA/IPAC Extragalactic Database NED which is operated by JPL CALTECH, under contract with NASA.

\section{References}

Acker, A., Marcout, J., Ochsenbein, F., Stenholm, B., \& Tylenda, R. 1992, Strasbourg-ESO catalogue of galactic planetary nebulae, Part $1 \& 2$

Ciardullo, R. 2003, in Stellar Candles for the Extragalactic Distance Scale, ed.

D. Alloin, \& W. Gieren, Lect. Notes Phys., 635, 243

Ciardullo, R., Jacoby, G. H., \& Ford, H. C. 1989a, ApJ, 344, 715

Ciardullo, R., Jacoby, G. H., Ford, H. C., \& Neill, J. D. 1989b, ApJ, 339, 53

Ciardullo, R., Feldmeier, J. J., Jacoby, G. H., et al. 2002, ApJ, 577, 31

Ciardullo, R., Durell, P. R., Laychack, M. B., et al. 2004, ApJ, 614, 167

Davidge, T. J. 1998, ApJ, 497, 650

Dopita, M. A., Jacoby, G. H., \& Vassiliadis, E. 1992, ApJ, 389, 27

Dopita, M. A., Vassiliadis, E., Wood, P. R., et al. 1997, ApJ, 474, 188

Durand, S., Dejonghe, H., \& Acker, A. 1996, A\&A, 310, 97

Feldmeier, J. J., Ciardullo, R., \& Jacoby, G. H. 1997, ApJ, 479, 231

Ferguson, Annette, M. N., Wyse, Rosemary, F. G., \& Gallagher, J. S. 1996, AJ, 112,2567

Fitzgibbons, G. L. 1990, Ph.D. Thesis, Florida Univ., Gainesville

Freedman, W. L., Madore, B. F., Hawley, S. L., et al. 1992, ApJ, 396, 80

Giuricin, G., Marinoni, C., Ceriani, L., \& Pisani, A. 2000, ApJ, 543, 178

Graham, J. A. 1982, ApJ, 252, 474

Grevesse, N., Noels, A., \& Sauval, A. J. 1996, in Cosmic Abundances, ed. S. S. Holt, \& G. Sonneborn (San Francisco: ASP), ASP Conf. Ser., 99, 117 Jacoby, G. H. 1989, ApJ, 339, 39

Jacoby, G. H., Quigley, R. J., \& Africano, J. L. 1987, PASP, 99, 672

Jacoby, G. H., Ciardullo, R., Ford, H. C., \& Booth, J. 1989, ApJ, 344, 70

Jacoby, G. H., Branch, D., Ciardullo, R., et al. 1992, PASP, 104, 599

Jacoby, G. H., \& Ciardullo, R. 1993, in Planetary Nebulae, ed. R. Weinberger, \& A. Acker (Kluwer), Proc. IAU Symp., 155, 503
Jerjen, H., Freeman, K. C., \& Binggeli, B. 1998, AJ, 116, 2873 Jerjen, H., Freeman, K. C., \& Binggeli, B. 2000, AJ, 119, 593

Karachentsev, I. D., Sharina, M. E., Grebel, E. K., et al. 2000, ApJ, 542, 128

Karachentsev, I. D., Grebel, E. K., Sharina, M. E., et al. 2003, A\&A, 404, 93

Magrini, L., Corradi, R. L. M., Greimel, R., et al. 2003, A\&A, 407, 51

Magrini, L., Perinotto, M., Mampaso, A., \& Corradi, R. L. M. 2004, A\&A, 426, 779

Mathieu, A., Dejonghe, H., \& Hui, X. 1996, A\&A, 309, 30

Monet, D., et al. 1998, PMM USNO-A2.0: A Catalogue of Astrometric Standards (Washington, DC: US Naval Obs.)

Napolitano, N. R., Arnaboldi, M., Freeman, K. C., \& Capaccioli, M. 2001, A\&A, 377,784

Pritchet, C. J., Schade, D., Richer, H. B., Crabtree, D., \& Yee, H. K. C. 1987, ApJ, 323, 79

Puche, D., \& Carignan, C. 1988, AJ, 95, 1025

Puche, D., Carignan, C., \& Wainscoat, R. J. 1991, AJ, 101, 447

Renzini, A., \& Buzzoni, A. 1986, in Spectral Evolution of Galaxies, ed. C. Chiosi, \& A. Renzini (Reidel), Ap. Space Sci. Lib., 122, 195

Schlegel, D. J., Finkbeiner, D. P., \& Davis, M. 1998, ApJ, 500, 525

Seaton, M. J. 1979, MNRAS, 187, 73

Soffner, T., Méndez, R. H., Jacoby, G. H., et al. 1996, A\&A, 306, 9

Tikhonov, N. A., Galazutdinova, O. A., \& Drozdovsky, I. O. 2005, A\&A, 431, 127

Tüllmann, R., Rosa, M. R., Elwert, T., et al. 2003, A\&A, 412, 69

Valdes, F. 1998, Guide to the NOAO mosaic data handling software

van den Bergh, S. 2000, PASP, 112, 529V

Walsh, J. R., Jacoby, G. H., Peletier, R. F., \& Walton, N. A. 2000, SPIE, Discoveries and Research Prospects from 8 to 10-Meter Class Telescopes, 4005, 131

Whiting, A. B. 1999, AJ, 117, 202

Zijlstra, A. A. 1999, in AGB stars, ed. T. Le Bertre, A. Lebre, \& C. Waelkens (San Francisco: ASP), IAU Symp., 191, 551 